\title{
Comparative study of thermostimulated luminescence and electron emission of nitrogen nanoclusters and films
}

\author{
R.E. Boltnev ${ }^{1}$, I.B. Bykhalo ${ }^{1}$, I.N. Krushinskaya ${ }^{1}$, A.A. Pelmenev ${ }^{1}$, V.V. Khmelenko ${ }^{2}$, \\ D.M. Lee ${ }^{2}$, I.V. Khyzhniy ${ }^{3}$, S.A. Uyutnov ${ }^{3}$, E.V. Savchenko ${ }^{3}$, A.N. Ponomaryov ${ }^{4}$, \\ G.B. Gumenchuk ${ }^{5}$, and V.E. Bondybey ${ }^{5}$ \\ ${ }^{1}$ Branch of Institute of Energy Problems of Chemical Physics RAS, Chernogolovka 142432, Russia \\ E-mail: boltnev@gmail.com \\ ${ }^{2}$ Department of Physics and Astronomy, Texas A\&M University, College Station, TX 77843, USA \\ ${ }^{3}$ B. Verkin Institute for Low Temperature Physics and Engineering of the National Academy of Sciences of Ukraine \\ 47 Lenin Ave., Kharkov 61103, Ukraine \\ ${ }^{4}$ Helmholtz Zentrum Dresden-Rossendorf, Dresden 01328, Germany \\ ${ }^{5}$ Lehrstuhl für Physikalische Chemie II TUM, Garching b. München 85747, Germany
}

Received December 5, 2012

\begin{abstract}
We have studied thermostimulated luminenscence and electron emission of nitrogen films and nanoclusters containing atomic nitrogen free radicals. Thermostimulated electron emission from $\mathrm{N}_{2}$ nanoclusters was observed for the first time. Thermostimulated luminescence spectra obtained during $\mathrm{N}_{2}-\mathrm{He}$ sample destruction are similar to those detected from $\mathrm{N}_{2}$ films pre-irradiated by an electron beam. This similarity reveals common mechanisms of energy transfer and relaxation. The correlation of the luminescence intensity and the electron current in both systems points to the important role of ionic species in relaxation cascades. A sublimation of solid helium shells isolating nitrogen nanoclusters is a trigger for the initiation of thermostimulated luminescence and electron emission in these nitrogen-helium condensates.
\end{abstract}

PACS: 78.60.Kn Thermoluminescence;

79.75.+g Exoelectron emission.

Keywords: solid helium, cryogenic electrolytes, ion dipole gas, phonon spectrum.

\section{Introduction}

Accumulation of reactive particles in nitrogen and nitrogen-containing solids has been a problem of particular interest for years [1-3]. A great breakthrough was made by use of a gas jet condensation technique [4,5] and the local concentrations of $\mathrm{N}$ atoms up to $10^{21} \mathrm{~cm}^{-3}$ were recently achieved in impurity-helium condensates (IHCs) [6]. IHCs are formed by impurity nanoclusters providing large total surface area favorable for stabilization of radicals. We have never registered any ions in impurity-helium condensates during previous ESR and optical spectroscopy studies. In this work we present the first observation of ions in IHCs and discuss our recent results of studies of the thermally stimulated luminescence (TSL) and electron emission
(TSEE) of nitrogen nanoclusters and films. Ionic nitrogen radicals were studied in solid films of neon [7-9] and molecular nitrogen [10]. Formation and relaxation of many excited complexes (including ionic ones) in solid films of rare gases and $\mathrm{N}_{2}$ have been well studied [11-13]. Moreover, crystals of solid ${ }^{4} \mathrm{He}$ (so-called "icebergs") doped by metal particles can store rather high density of ions $\sim 10^{14}-10^{15} \mathrm{~cm}^{-3}$ [14]. Such icebergs remain solid below the melting curve of ${ }^{4} \mathrm{He}$. This observation gives us a facinating example of metastable Coulomb systems in condensed helium. A deep understanding of relaxation processes involving both neutral and charged species in such energetic materials as IHCs can provide for further advances in energy accumulation in cryogenic media. 


\section{Experimental setups}

A gas jet condensation technique [4,5] was used to accumulate nitrogen nanoclusters in superfluid helium. A gas mixture of $\mathrm{N}_{2}(1 \%)$ and $\mathrm{He}$ was transported from a room temperature gas handling system to the cryogenic region. Nitrogen atoms were produced by dissociation of $\mathrm{N}_{2}$ molecules by using a high-power radiofrequency (RF) discharge $(f \sim 40 \mathrm{MHz}, P \sim 40 \mathrm{~W})$ applied to electrodes placed around the quartz capillary carrying the mixed gases. The resulting jet of helium gas with nitrogen particles emerging from the quartz capillary was directed onto the surface of superfluid ${ }^{4} \mathrm{He}$ contained in a glass beaker. A fountain pump situated in the bottom part of the optical glass dewar maintained a constant liquid helium level in the beaker. During the cooling of the gas jet, at first nitrogen nanoclusters are formed. When the temperature gets lower, helium atoms are adsorbed on the surface of the nitrogen clusters. The temperature during sample preparation was about $1.5 \mathrm{~K}$. As the jet penetrated the surface of the liquid helium, a macroscopic snow-like translucent material consisting of nitrogen nanoclusters isolated by solid helium shells was created. A jet with a flux of $\sim 5 \cdot 10^{19}$ atoms and molecules per second yielded $\sim 0.3-0.4 \mathrm{~cm}^{3}$ of sample in 10 min. Warming the sample when removed from bulk liquid helium initiates evaporation of helium layers, giving rise to direct contact between neighboring cluster surfaces, accompanied by recombination of the reactive species. During the sample warm-up the TSL spectra were repeatedly detected with an Ocean Optics spectrometer HR2000+ in the range 200-1100 nm with the exposure time of $1 \mathrm{~s}$. The glass dewars and beaker restricted the accessible spectral range between 325 and $1100 \mathrm{~nm}$. The optical resolution of the spectrometer was of order $1.3 \mathrm{~nm}$ (FWHM). For the first time we observed electrical currents accompanying the destruction of nitrogen-helium samples collected on an electrode held at the positive potential $(9 \mathrm{~V})$. A signal collected by the electrode was digitized with a picoammeter (Keithley 6485).

In another series of experiments, solid nitrogen films were grown in two ways: by deposition of nitrogen gas under an electron beam or by deposition of neutral gas which was then irradiated with an electron beam of a given energy, which can be varied from $500 \mathrm{eV}$ to $2 \mathrm{keV}$. The high-purity (99.9995\%) gas was deposited on a metal substrate cooled to $6 \mathrm{~K}$ in a vacuum chamber with the base pressure $10^{-8}$ mbar. The presence of impurities was monitored spectroscopically. The main impurity appeared to be oxygen. The resulting condensates were polycrystalline films of $100 \mu \mathrm{m}$ thickness. Note that the samples with an open surface are accessible to luminescence studies in the temperature range up to $36 \mathrm{~K}$, the range of existence of the $P a 3$ structure ( $\alpha$ phase). Transition to the $\beta$ phase at $35.6 \mathrm{~K}$ in such films was detected previously by the TSEE technique [13]; however the intense sublimation of nitro- gen films limits studies of their spectroscopy at higher temperatures. The luminescence spectra in these experiments were also recorded repeatedly for the exposure time required to monitor the formation and accumulation of excited species. On completion of irradiation, afterglow and "afteremission" of electrons were detected. When these effects decayed completely, samples of solid $\mathrm{N}_{2}$ were heated at a constant rate. The temperatures required for sample preparation and the heating rate were controlled with a silicon diode sensor mounted at the substrate which was connected to a temperature controller (Leybold LTC 60). Yields of spectrally resolved thermally stimulated luminescence and exoelectron current were detected in a time-correlated fashion. The TSL spectra in the range 170-870 $\mathrm{nm}$ were recorded using an Ocean Optics spectrometer S2000, the TSEE current was detected with an electrode kept at $+9 \mathrm{~V}$, and amplified by a FEMTO DLPCA 200 current amplifier. More detailed descriptions of the experimental setup can be found elsewhere $[15,16]$.

\section{Experimental results}

The time dependences of the current, the temperature and the integrated (in the range from 300 up to $1100 \mathrm{~nm}$ ) luminescence intensity obtained during warm-up and destruction of nitrogen-helium sample are shown in Fig. 1. In the case of highly porous impurity-helium sample, a warmup of its different parts is not uniform due to poor thermal conductivity and there is no direct relation between the thermometer temperature and the temperature of the reacting part (which emits light). We can only estimate the temperature of the complete sample destruction (when the last part of the sample is burned out). This temperature is about $12 \mathrm{~K}$ (Fig. 1). We have missed some current counts at the beginning of the warm-up due to a late start of picoammeter software. Nevertheless one can see a correlation between the current and the integrated luminescence intensity. This means that the main trigger for nitrogen-helium sample

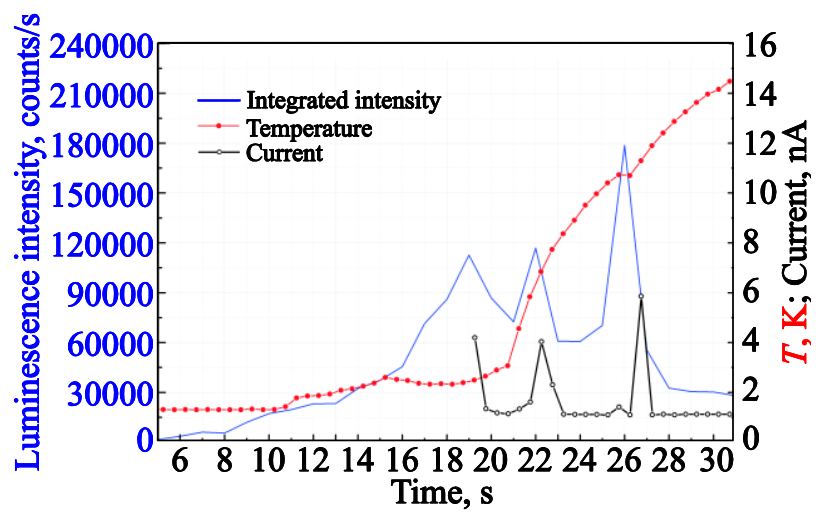

Fig. 1. (Color online) The time dependence of the current (black line with hollow circles), the integrated luminescence intensity (blue line), and the temperature (red line with solid circles) detected during warm-up and destruction of a nitrogen-helium sample. 


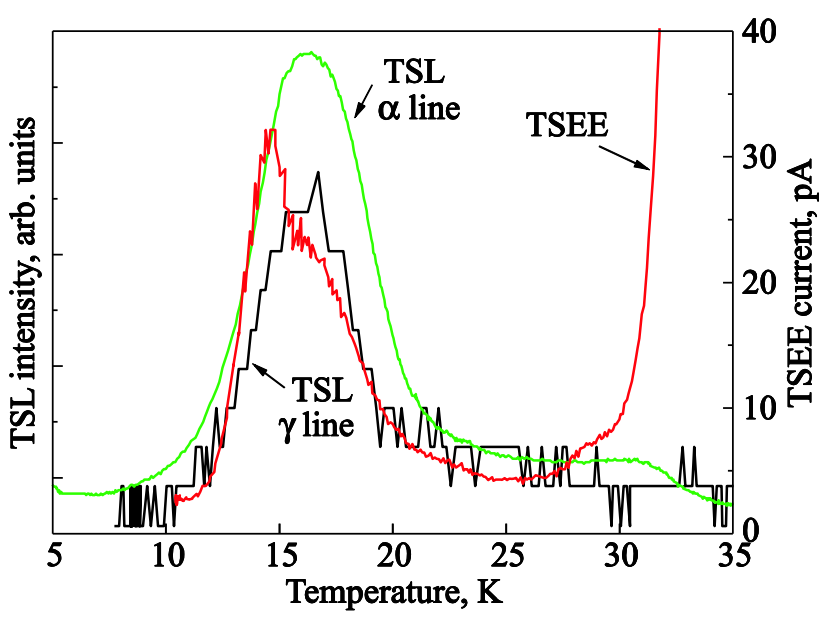

Fig. 2. The thermostimulated electron emission and the luminescence intensities at 522 and $793 \mathrm{~nm}$ of a solid nitrogen film preirradiated with a $1 \mathrm{keV}$ electron beam.

destruction is the evaporation of helium atoms from the impurity nanocluster surfaces which occurs for temperatures above $2 \mathrm{~K}$. If energetic nitrogen-helium samples with high-energy content warmed up at low pressure (less than $500 \mathrm{~Pa}$ ) they sublimated at temperatures below 12-14 K. Some delay of the current peaks relative to the luminescence flashes can be explained by thermo- and photostimulated mechanisms of electron de-trapping, when the intense recombination of $\mathrm{N}$ atoms stabilized mainly on the cluster surfaces produces a bright emission and accelerates the sample warming. As demonstrated in [17,18], the electron emission from RG solids containing charge centers can be stimulated by both irradiation with photons and sample warming.

The recent observations of the TSEE from solid nitrogen [13] provide a new means for investigating the relaxation processes occurring in solid nitrogen by simultaneously using the optical and current activation spectroscopy methods. As mentioned above, after completion of the sample irradiation the well-known [19] long lived afterglow (the so-called $\alpha$ group) at the wavelength of the ${ }^{2} D \rightarrow{ }^{4} S$ doubly forbidden transition of $\mathrm{N}$ atom $(522 \mathrm{~nm})$ appears, with a characteristic decay time $\tau$ of about $37 \mathrm{~s}$. We observed also a persistent after-emission of electrons exhibiting two exponential decays, one with $\tau_{1}=37 \mathrm{~s}$, coinciding with the afterglow decay time $\tau$, and the another with a longer $\tau_{2}$ about $215 \mathrm{~s}$.

Before starting the warm-up experiments and recording the "glow curves", both the afterglow and the after-emission were allowed to drop to zero, indicating that all the excited $\mathrm{N}$ atoms produced under irradiation had sufficient time to return to the ground state. The actual curves showing the temperature dependence of the TSEE and the spectrally resolved thermally stimulated luminescence TSL over the range of the $\alpha$ phase existence obtained during the warm-up of a nitrogen film are presented in Fig. 2, where

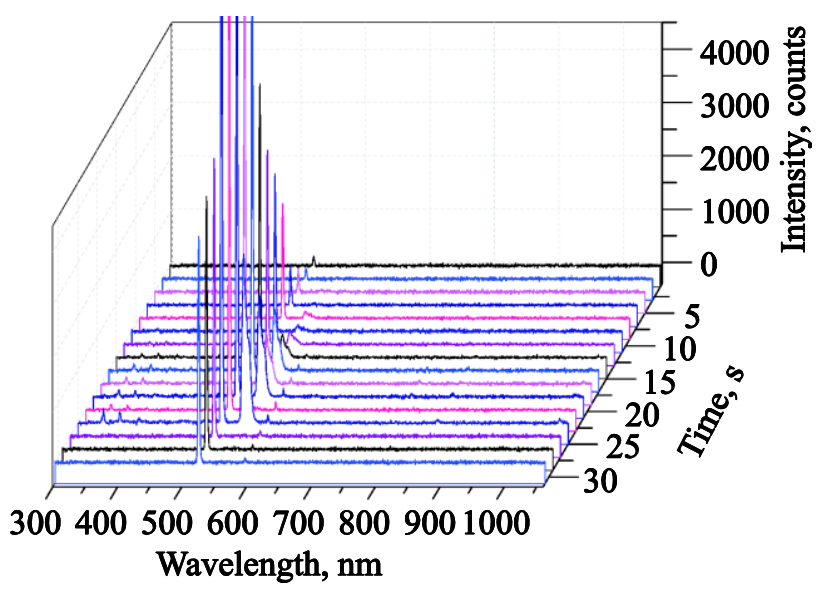

Fig. 3. Dynamics of thermoluminescence spectra of a nitrogenhelium sample. Every 2nd of the spectra measured is shown.

for the TSL curve, the $\alpha$ group being the most intense line was monitored. The TSL shows a wide feature with maximum peaked at $16 \mathrm{~K}$ and a "high temperature" shoulder which extends up to $30 \mathrm{~K}$. The TSEE curve at low temperatures consists of two overlapping peaks at 15 and $16 \mathrm{~K}$ with an extended "high temperature" tail. The TSEE yield exhibits a sharp increase as the phase transition to the $\beta$ phase is approached. Besides the $\alpha$ group, we also monitored the TSL at $793 \mathrm{~nm}$, the wavelength of the so-called " $\gamma$ line" - an unidentified line. The similarity of the glow curves detected at 793 and $522 \mathrm{~nm}$ shown in Fig. 2 implies a similar mechanism for a population of the emitting states. Elucidation of the processes resulting in $\gamma$-line emission requires further study.

The dynamics of TSL spectra during warm-up of a nitrogen-helium sample and a "pure" solid nitrogen film are shown in Figs. 3 and 4, respectively.

We should note that reappearance of the 522.7 and the $555.5 \mathrm{~nm}$ lines in the TSL spectra from a nitrogen solid film was not reproducible (Fig. 4). In fact, we observed

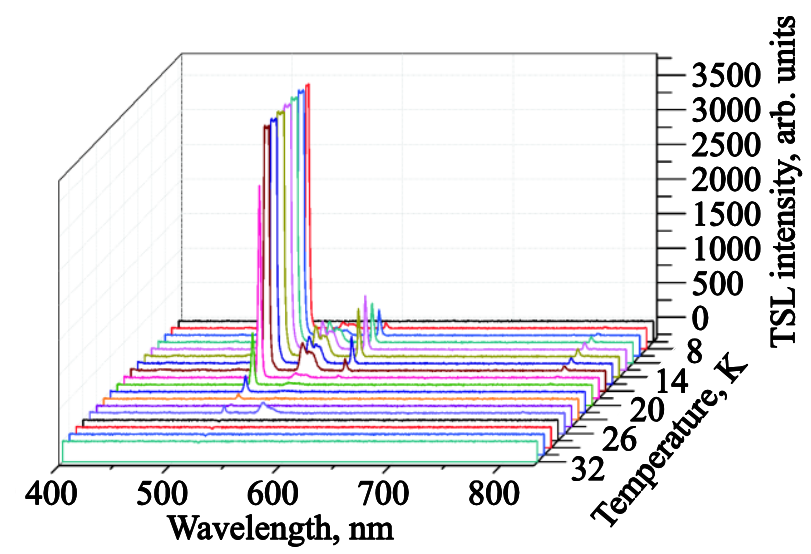

Fig. 4. Dynamics of thermoluminescence spectra of a solid nitrogen film. Every 20th of the spectra measured is shown. 


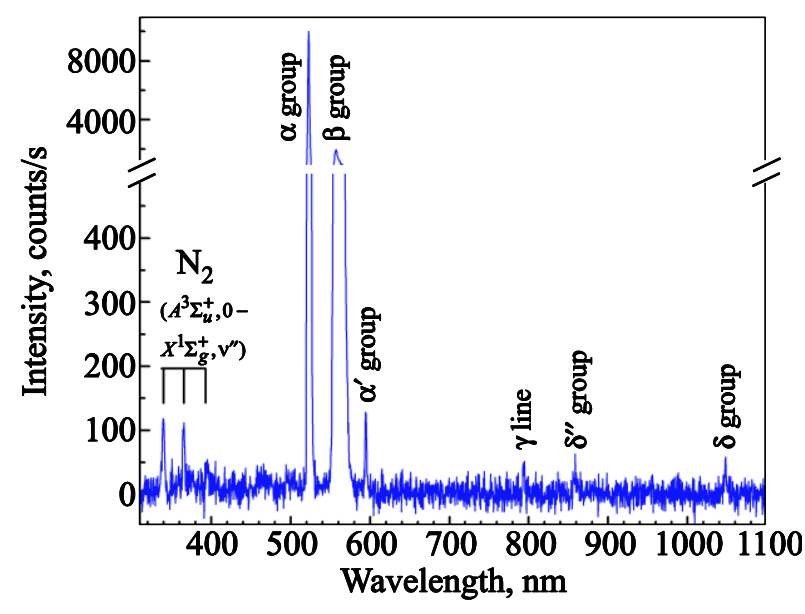

Fig. 5. The most intense flash spectrum detected during the nitrogen-helium sample destruction.

some flashes in samples and films containing minor admixture of oxygen. The similar effect was mentioned in earlier work, e.g., [20].

The most intense flash spectrum detected during the nitrogen-helium sample destruction is shown in Fig. 5. Various different states of atomic and molecular nitrogen along with those of atomic oxygen involved in the energy relaxation processes. The $\alpha$ and $\beta$ groups corresponding to the transitions $\mathrm{N}\left({ }^{2} D \rightarrow{ }^{4} S\right)$ and $\mathrm{O}\left({ }^{1} S \rightarrow{ }^{1} D\right)$ dominate the spectrum. Weak Vegard-Kaplan bands of $\mathrm{N}_{2}$ molecules, the $\alpha^{\prime}$, $\delta$, and $\delta^{\prime \prime}$ groups of $\mathrm{N}$ atoms, and the $\gamma$ line are also shown in the spectrum.

The typical TSL spectrum detected during a warm-up of the solid nitrogen film pre-irradiated with an electron beam is shown in Fig. 6. The spectrum is rather similar to the one shown in Fig. 5. The spectral features of the TSL observed from the $\mathrm{N}_{2}-\mathrm{He}$ sample and the $\mathrm{N}_{2}$ film together with their identifications are summarized in Table 1.

The positions of the spectral features observed are close to the ones known for $\mathrm{N}_{2}$ molecules, $\mathrm{N}$ and $\mathrm{O}$ atoms in $\mathrm{N}_{2}$

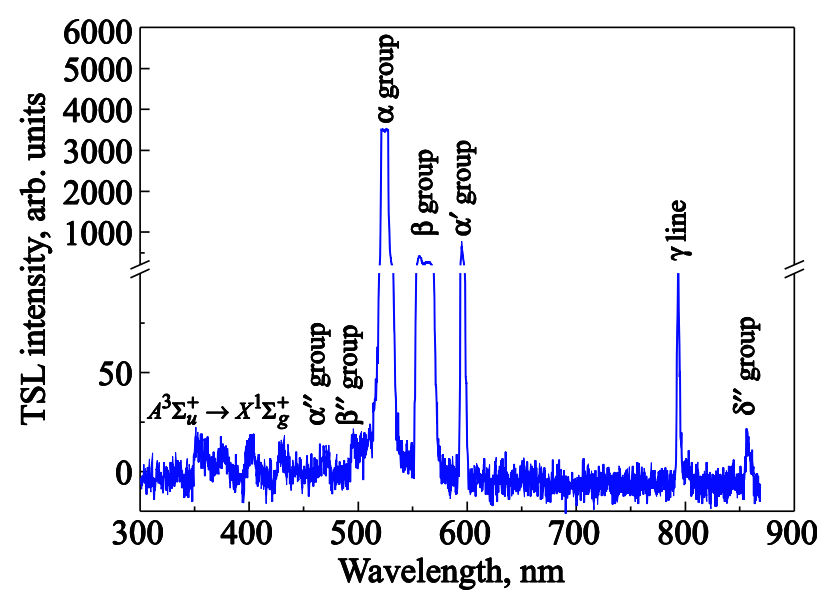

Fig. 6. The TSL spectrum detected at $16 \mathrm{~K}$ during the solid nitrogen film warm-up. matrices. Keeping in mind the spectral resolution of the spectrometers used in these studies, the agreement of the results obtained with clusters and solid films with the published data on emission from $\mathrm{N}_{2}$ matrices is quite satisfactory.

Table 1. The spectral features of TSL observed from the $\mathrm{N}_{2}-\mathrm{He}$ sample and the $\mathrm{N}_{2}$ film, their identification and positions in comparison with literature data

\begin{tabular}{|c|c|c|c|}
\hline 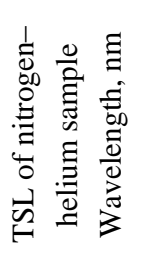 & 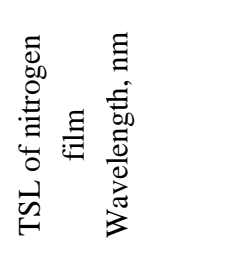 & 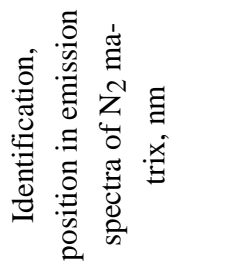 & 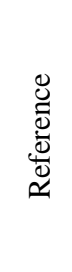 \\
\hline $\begin{array}{l}339.6 \\
365.8 \\
395.3 \\
\end{array}$ & $\begin{array}{c}\text { (very weak and } \\
\text { broad) }\end{array}$ & $\begin{array}{c}\text { bands of Vegard- } \\
\text { Kaplan system: } \\
0-9, \quad 338.6 \\
0-10,364.4 \\
0-11,395 \\
\end{array}$ & [21] \\
\hline 522.6 & 522.7 & $\begin{array}{c}\alpha \text { group, } \\
\mathrm{N}\left({ }^{2} D \rightarrow 4 S\right), 523 \\
\end{array}$ & [20] \\
\hline 557.4 & 555.5 & $\begin{array}{c}\beta \text { group, } \\
\mathrm{O}\left({ }^{1} S \rightarrow 1 D\right), 554.2\end{array}$ & [20] \\
\hline 594.4 & 594 & $\alpha^{\prime}$ group, 594.5 & [20] \\
\hline 794.4 & 792.6 & $\begin{array}{c}\gamma \text { line, } 793.5 \\
\text { (upon irradiation) }\end{array}$ & [20] \\
\hline 858.5 & 855.1 & $\delta^{\prime \prime}$ group, 856 & [20] \\
\hline 1047.7 & $\begin{array}{l}\text { beyond the oper- } \\
\text { ating range of the } \\
\text { spectrometer }\end{array}$ & $\begin{array}{c}\delta \text { group, } \\
\mathrm{N}\left({ }^{2} P \rightarrow^{2} D\right), 1045\end{array}$ & [20] \\
\hline
\end{tabular}

\section{Discussion}

Generaly, the TSL spectra obtained during the $\mathrm{N}_{2}-\mathrm{He}$ sample warm-up are similar to those detected from the $\mathrm{N}_{2}$ films, while the final destruction temperature of nitrogenhelium sample is close to the temperature at which the TSEE and TSL start in nitrogen films. In both systems we observed strong the $\alpha$ and $\beta$ groups, the $\alpha^{\prime}$ and $\delta^{\prime \prime}$ groups of $\mathrm{N}$ atoms, an unidentified $\gamma$ line and Vegard-Kaplan bands of $\mathrm{N}_{2}$ molecules. It means that similar energy transfer and relaxation channels are involved in both systems studied. Some small quantitative differences observed in the spectra can be easily understood. For example, the more intense $\beta$ group observed in the TSL spectrum of the nitrogen-helium sample (Fig. 5) can be simply explained by the higher oxygen content in the sample. Close inspection of the TSL dynamics of nitrogen films shows that the ratio of the intensities of $\alpha$ and $\alpha^{\prime}$ groups, $I_{\alpha} / I_{\alpha^{\prime}}$ (about 70), is somewhat smaller than that in nitrogen-helium samples $\left(I_{\alpha} / I_{\alpha^{\prime}}=80\right)$ [5]. The intensity of the $\beta$ group emission of $\mathrm{O}$ atoms is comparable with the intensity of $\alpha$ group emissions of $\mathrm{N}$ atoms because of the much higher $\left(\sim 10^{7}\right.$ times $)$ 
probability of the $\mathrm{O}\left({ }^{1} S \rightarrow{ }^{1} D\right)$ transition as compared with the $\mathrm{N}\left({ }^{2} D \rightarrow{ }^{4} S\right)$ transition. The main source of oxygen in nitrogen-helium samples are different impurities such as $\mathrm{O}_{2}, \mathrm{OH}, \mathrm{H}_{2} \mathrm{O}$ in gaseous helium giving totally $\sim 10 \mathrm{ppm}$. Although the concentration of stabilized $\mathrm{N}$ atoms in solid $\mathrm{N}_{2}$ matrix is three orders of magnitude larger than that of the $\mathrm{O}$ atoms, the significantly larger transition probability of $\mathrm{O}$ atoms leads to a noticeable emission from the $\mathrm{O}$ atoms. An oxygen impurity also appears in the nitrogen film studies from residual gases in the deposition chamber especially at low gas flow rate.

It is notable that the TSEE currents detected for both systems correlated with the corresponding TSL signals, pointing to the important role of the ionic species in the relaxation cascades. The intense release of negative charge carriers from the nitrogen-helium sample beginning at temperatures below $8-10 \mathrm{~K}$ indicates that electrons are the best candidates to be the charge carriers. The observation of three electron emission peaks (see Fig. 1) may lead to the destruction of different regions of the impurity-helium sample because of its inhomogeneous warm-up, which releases the electrons from these regions. Alternatively, the observed peaks may be explained by the existence of a corresponding number of electron traps with different activation energies. The lowest temperature peak may be related to the release of electrons from "bubbles" destroyed during liquid helium evaporation. As is known, electrons are self-trapped in liquid helium via the "bubble" structure formation [22].

In the TSL of nitrogen films a broad main peak at $16 \mathrm{~K}$ with an extended "high temperature" shoulder dominates the TSL glow curve. This suggests a relatively wide distribution of trap depths and activation energies for stored charges. An average activation energy deduced by the halfwidth method [23] for traps which form the $16 \mathrm{~K}$ peak is about $14 \mathrm{meV}$. Close correlation of the TSL detected for the $\alpha$ group and TSEE current at temperatures up to $25 \mathrm{~K}$ suggests that this luminescence arises from the neutralization reaction of the ionic species $\mathrm{N}^{+}$with electrons detrapped upon the warm-up of the nitrogen film: $\mathrm{N}^{+}+\mathrm{e}^{-} \rightarrow \mathrm{N}^{*}$. This appears plausible taking into account that the ionization potential of the $\mathrm{N}$ atom is $14.53 \mathrm{eV}$ is lower than that for nitrogen molecule $(15.58 \mathrm{eV})$. Despite the firm data on TSEE from solid nitrogen films [13] there is still an interesting question regarding the presence and specific nature of positive ions. Molecular ions $\mathrm{N}_{2}^{+}$and $\mathrm{N}_{4}^{+}$had been detected by ESR spectroscopy [7,8] and IR absorption [9] in neon matrices. These ions were formed in the neon matrix by electron bombardment and photoionization. Note that they were detected only when $\mathrm{N}_{2} / \mathrm{Ne}$ ratio was greater than $1 / 600$ and a quenching reactant was codeposited with the nitrogen [8]. Up to now, in our ESR and optical spectroscopy studies we did not observe the ion presence in either nitrogen-helium or nitrogen-neon-helium samples. However quite recently the presence of $\mathrm{N}_{4}^{+}$in the electron bombarded solid nitrogen films was revealed using current and optical activation spectroscopy in the VUV range [10]. The tetranitrogen cation $\mathrm{N}_{4}^{+}$manifested itself by the dissociative recombination reaction with an electron released from the trap upon warm-up:

$$
\mathrm{N}_{4}^{+}+\mathrm{e} \rightarrow \mathrm{N}_{2}+\mathrm{N}_{2}^{*}\left(a^{\prime} \Sigma_{u}^{-}\right) \rightarrow \mathrm{N}_{2}+\mathrm{N}_{2}+h v(\mathrm{VUV})
$$

An application of the RF discharge almost excludes the possibility of trapping predominantly the charges of one sign in impurity-helium condensates. The origin as well as the specific nature of ions in impurity-helium condensates produced by RF discharge is still unclear. There are two main possibilities for creating ions in IHCs: (1) during a gas jet condensation, ions formed in an RF discharge region (possibly, due to deep UV irradition of the clusters from the helium gas discharge) can be trapped by growing impurity clusters (moreover, they themselves can be nucleation centers due to the higher polarizability); (2) the ionization of particles during the sample destruction due to interaction of excited atoms and molecules following the recombination of stored radicals. The first possibility appears more preferred because of our spectroscopic observation of $\mathrm{N}_{2}^{+}$ions in the condensing gas jet [24]. It is now well known that sublimation of solid helium shells isolating the impurity nanoclusters triggers the recombination of radicals stabilized on surfaces of the clusters and gives rise to the thermostimulated luminescence $[5,25]$. The electron emission from nitrogen-helium condensates at the same time as the TSL suggests a similar allocation of electrons, on the surfaces or in outer layers of the nitrogen clusters.

\section{Conclusions}

1. Thermostimulated electron emission in nitrogenhelium condensates during their destruction was observed for the first time.

2 . The same energy relaxation pathways were thermally initiated in nitrogen nanoclusters and films prepared by different techniques. The earlier start (at lower temperatures) of the thermoluminescence and the electron emission from nitrogen nanoclusters is determined by intense recombination of nitrogen atoms stabilized on clusters' surfaces following evaporation of solid helium shells surrounding the clusters.

3. Thermostimulated luminescence and electron emission observed during warm-up of nitrogen-helium condensates and nitrogen films demonstrate the similarity of the processes initiated, including the neutralization of the ionic species.

4. The electron emission from nitrogen-helium samples upon their destruction can be explained either by a capture of ions in nitrogen nanoclusters during the sample preparation or by the creation of the ions as a result of interaction of excited atoms and molecules during the sample destruction. 
The study was supported by grants No. 010366-0137-2009 Norman Hackerman Advanced Research Program, NSF No. DMR 1209255, RFBR Nos. 11-02-92500-CRDF-E_a and 11-08-01232-a, CRDF No. RUP1-7025-CG-11, federal contract 11.519.11.6021 with Russian Federation Ministry of Education and Science. E.S., I.Kh. and S.U. thank Deutsche Forschungsgemeinschaft for the travel grant.

1. A.M. Bass and H.P. Broida, Formation and Trapping of Free Radicals, Academic Press, New York (1960).

2. P.C. Samartzis and A.M. Wodtke, Intern. Rev. Phys. Chem. 25, 527 (2006).

3. Y. Shi and D.W. Brenner, J. Chem. Phys. 127, 134503 (2007).

4. E.B. Gordon, L.P. Mezhov-Deglin, and O.F. Pugachev, JETP Lett. 19, 103 (1974).

5. V.V. Khmelenko, I.N. Krushinskaya, R.E. Boltnev, I.B. Bykhalo, A.A. Pelmenev, and D.M. Lee, Fiz. Nizk. Temp. 38, 871 (2012) [Low Temp. Phys. 38, 688 (2012)].

6. S. Mao, R.E. Boltnev, V.V. Khmelenko, and D.M. Lee, Fiz. Nizk. Temp. 38, 1313 (2012) [Low Temp. Phys. 38, 1037 (2012)].

7. L.B. Knight, Jr., J.M. Bostick, R.W. Woodward, and J. Steadman, J. Chem. Phys. 78, 6415 (1983).

8. L.B. Knight, Jr., K.D. Johannessen, D.C. Cobranchi, E.A. Earl, D. Feller, and E.R. Davidson, J. Chem. Phys. 87, 885 (1987).

9. M.E. Jacox, Research on Chemical Intermediates 12, 33 (1989).

10. E.V. Savchenko, I.V. Khyzhniy, S.A. Uyutnov, G.B. Gumenchuk, M.K. Beyer, A.N. Ponomaryov, and V.E. Bondybey, will be submitted to J. Chem. Phys.

11. G. Gumenchuk, A. Ponomaryov, I. Khyzhniy, S. Uyutnov, E. Savchenko, and V. Bondybey, Phys. Proc. 2, 441 (2009).
12. E.V. Savchenko, I.V. Khyzhniy, S.A. Uyutnov, G.B. Gumenchuk, A.N. Ponomaryov, and V.E. Bondybey, Fiz. Nizk. Temp. 35, 520 (2009) [Low Temp. Phys. 35, 409 (2009)].

13. I. Khyzhniy, E. Savchenko, S. Uyutnov, G. Gumenchuk, A. Ponomaryov, and V. Bondybey, Rad. Meas. 45, 353 (2010).

14. P. Moroshkin, V. Lebedev, and A. Weis, Phys. Rev. Lett. 102, 115301 (2009).

15. A.N. Ponomaryov, E.V. Savchenko, I.V. Khyzhniy, G.B. Gumenchuk, M. Frankowski, and V.E. Bondybey, Fiz. Nizk. Temp. 33, 705 (2007) [Low Temp. Phys. 33, 532 (2007)].

16. A. Ponomaryov, G. Gumenchuk, E. Savchenko, and V.E. Bondybey, Phys. Chem. Phys. 9, 1329 (2007).

17. E.V. Savchenko, I.V. Khizhniy, G.B. Gumenchuk, A.N. Ponomaryov, and V.E. Bondybey, Fiz. Nizk. Temp. 32, 1417 (2006) [Low Temp. Phys. 32, 1078 (2006)].

18. E.V. Savchenko, A.G. Belov, G.B. Gumenchuk, A.N. Ponomaryov, M.K. Beyer, V.E. Bondybey, and M. Frankowski, Rad. Phys. \& Chem. 76, 577 (2007).

19. A. Lofthus and P.H. Krupenie, J. Phys. Chem. Ref. Data 6, 113 (1977).

20. M. Peyron, E.M. Hörl, H.W. Brown, and H.P. Broida, J. Chem. Phys. 30, 1304 (1959).

21. Yu.B. Poltoratski and I.Ya. Fugol, Fiz. Nizk. Temp. 5, 925 (1979) [Sov. J. Low Temp. Phys. 5, 439 (1979)].

22. J. Jortner, N.R. Kestner, S.A. Rice, and M.H. Cohen, J. Chem. Phys. 43, 2614 (1965).

23. D.R. Vij, Thermoluminescence, in: Luminescence of Solids, D.R. Vij (ed.), Plenum Press, New York (1998), p. 271.

24. E.B. Gordon, M.V. Martynenko, A.A. Pelmenev, O.F. Pugachev, and V.V. Khmelenko, Khim. Fiz. 13, 15 (1994).

25. V.V. Khmelenko, A.A. Pelmenev, I.N. Krushinskaya, I.B. Bykhalo, R.E. Boltnev, and D.M. Lee, Accepted for publication in J. Low Temp. Phys., DOI: 10.1007/s10909-012-0707-3. 